\title{
The clinical suspicion of a leaking intrathoracic esophagogastric anastomosis: the role of $\mathrm{CT}$ imaging
}

\author{
Victor D. Plat ${ }^{1}$, Boukje T. Bootsma ${ }^{1}$, Jennifer Straatman ${ }^{1}$, Janneke van den Bergh ${ }^{2}$, \\ Jan-Hein T. M. van Waesberghe ${ }^{2}$, Joanna Luttikhold ${ }^{1}$, Micha D. P. Luyer ${ }^{3}$, Donald L. van der Peet ${ }^{1}$, \\ Freek Daams ${ }^{1}$
}

${ }^{1}$ Department of Gastrointestinal Surgery, Amsterdam UMC, VU University Medical Center, Amsterdam, The Netherlands; ${ }^{2}$ Department of Radiology, Amsterdam UMC, VU University Medical Center, Amsterdam, The Netherlands; ${ }^{3}$ Department of Gastrointestinal Surgery, Catharina Hospital, Eindhoven, The Netherlands

Contributions: (I) Conception and design: VD Plat, J Straatman, JHTM van Waesberghe, DL van der Peet, F Daams; (II) Administrative support: None; (III) Provision of study materials or patients: None; (IV) Collection and assembly of data: All authors; (V) Data analysis and interpretation: VD Plat, BT Bootsma, J Straatman; (VI) Manuscript writing: All authors; (VII) Final approval of manuscript: All authors.

Correspondence to: Mr. Victor Dirk Plat, MD. Department of Gastrointestinal Surgery, VU University Medical Center, De Boelelaan 1117, ZH 7F020, 1081 HV Amsterdam, The Netherlands. Email: V.plat@amsterdamumc.nl.

Background: CT imaging is the primary diagnostic approach to assess the integrity of the intrathoracic anastomosis following Ivor Lewis esophagectomy. In the postoperative setting interpretation of CT findings, such as air and fluid collections, may be challenging. Establishment of a scoring system that incorporates CT findings to diagnose anastomotic leakage could assist radiologists and surgeons in the postoperative phase.

Methods: Consecutive patients who underwent a CT scan for a clinical suspicion of postoperative anastomotic leakage following Ivor Lewis esophagectomy between 2010 and 2016 in two medical centers were retrospectively included. Scans were excluded when oral contrast was not (correctly) administered. Acquired images were randomized and independently assessed by two experienced gastrointestinal radiologists, blinded for clinical information. For this study anastomotic leakage was defined as a visible defect during endoscopy or thoracotomy.

Results: A total of 80 patients had 101 CT scans, resulting in 32 scans with a confirmed anastomotic leak (25 patients). After multivariable backward stepwise logistic regression, a practical 5 -point scoring system was developed, which included the following CT findings: presence of extraluminal oral contrast, air collection at the anastomotic site, fluid collection at the anastomotic site, pneumothorax and loculated pleural effusion. Patients with a score of $\geq 3$ were considered at high risk for anastomotic leakage (positive predictive value: $83.3 \%$ ), patients with scores $<3$ were considered at low risk for anastomotic leakage (negative predictive value: $84.4 \%$ ). The scoring system showed a superior diagnostic performance compared to the original CT report and blinded interpretation of two radiologists.

Conclusions: Our CT-based practical scoring system enables a standardized approach in CT assessment and could facilitate early recognition of anastomotic leakage in patients after Ivor Lewis esophagectomy.

Keywords: Esophagectomy; anastomotic leakage; computed tomography; scoring system; esophageal cancer

Submitted Mar 04, 2020. Accepted for publication Aug 19, 2020.

doi: $10.21037 /$ jtd-20-954

View this article at: http://dx.doi.org/10.21037/jtd-20-954 


\section{Introduction}

Esophageal cancer is the 8th most common malignancy and 6th leading cause of cancer-related mortality worldwide. Annually, more than 450,000 new esophageal cancer cases and 400,000 cancer deaths are estimated (1). Trimodality therapy, consisting of neoadjuvant chemoradiotherapy and esophagectomy, represents the gold standard for potentially curable esophageal cancer (2). Esophagectomy with the creation of an intrathoracic anastomosis is increasingly applied (3) and might reduce anastomotic leakage incidence compared to a cervical anastomosis (4). In the Netherlands, the incidence of intrathoracic anastomotic leakage is approximately $18 \%$ and remains the primary source of morbidity and mortality $(5,6)$. Anastomotic leakage is frequently accompanied by other complications and adversely impact survival rates (7), recurrence of disease (8) and is associated with an extended postoperative period, leading to a substantial increase in hospital costs $(9,10)$. These adverse effects may be averted if anastomotic leakage is diagnosed early and intervened properly.

Clinical presentations of anastomotic leakage are diverse and varies from asymptomatic to severely septic patients. Clinical distinction between anastomotic leakage and other complications such as pneumonia and systemic inflammatory response syndrome (SIRS) is challenging as clinical symptoms are near to identical $(11,12)$. Therefore, leakage is mainly detected using a combination of clinical, endoscopic and radiological evaluation. Assessment of thoracic computed tomography (CT) images, especially during the early postoperative period, may be challenging. The presence of mediastinal air or fluid collections may indicate leakage out of the gastric conduit, but may also be a normal postoperative finding (13). These challenges are also encountered following other gastrointestinal surgical procedures and previous studies have found several radiological findings useful for the prediction of anastomotic leakage following colorectal, pancreatic and gastric surgery $(14,15)$.

Objective of this study was to determine the diagnostic value of CT in case of suspected anastomotic leakage. A secondary objective was to identify reliable CT findings predicting anastomotic leakage. Based on these findings a practical scoring system was proposed in order to aid radiologists and surgeons in the systematic assessment of CT scans. This scoring system may facilitate objective interpretation of CT findings to confirm or rule out anastomotic leakage.

\section{Methods}

\section{Study population}

This study was conducted in accordance with the Declaration of Helsinki (as revised in 2013). The study protocol was approved by the Medical Ethical Review Committee of the VU University Medical Center (2016.480) and by the local institutional ethical review board of the Catharina Hospital.

This study was designed as a multicenter retrospective cohort study. All consecutive patients underwent a CT scan for a clinical suspicion of anastomotic leakage following Ivor Lewis (16) esophagectomy between October 2010 and July 2016 in the VU University Medical Center in Amsterdam or in the Catharina Hospital in Eindhoven. The surgical procedure consisted of a two-stage laparoscopic and thoracoscopic esophagectomy with enbloc lymphadenectomy, the formation of a gastric conduit and the creation of a thoracic esophagogastric anastomosis. The anastomosis was created in an end-to-side fashion using $25 \mathrm{~mm}$ circular stapling or end-to-side/side-to-side using linear stapling and sutured closure of the stapling defect. CT imaging was performed if patients were suspect of anastomotic leakage within the first 30 days after surgery, based on clinical examination of the patient and laboratory findings. To prevent bias in assessment of leakage of contrast, scans were excluded when oral contrast was not (correctly) admitted. This study was reported according to The Strengthening the Reporting of Observational Studies in Epidemiology (STROBE) reporting checklist (17) (available at http://dx.doi.org/10.21037/jtd-20-954).

\section{Image acquisition}

Thoraco-abdominal CT images were acquired using commercially available 16-, 64- or 128-section CT scanners (Philips Medical Systems, Best, The Netherlands and Siemens, Erlangen, Germany). Images were typically acquired with $16 \times 0.9,64 \times 0.625$ or $128 \times 0.625 \mathrm{~mm}$ section collimation, a tube rotation time of $330-750 \mathrm{~ms}$, a tube potential of 100 or $120 \mathrm{kV}$, an effective tube current ranging from of $20-1,000 \mathrm{mAs}$ and a pitch of $0.7,0.9$ or 1.1 . An iodinated $70-100 \mathrm{~mL}$ contrast material bolus $(300 \mathrm{mg} / \mathrm{mL})$ was administered intravenously at 2 to $4 \mathrm{~mL} / \mathrm{sec}$ in all patients. Scan delay ranged from $25 / 30$ and $60 / 70$ seconds after injection of contrast. Patients received $200 \mathrm{~mL}$ iodinated oral contrast media (Telebrix Gastro, $300 \mathrm{mgI} / \mathrm{mL}$ ) 
in the first hour prior to CT imaging, followed by $200 \mathrm{~mL}$ just five minutes before imaging.

\section{Parameter selection and definitions}

CT findings of interest were selected prior to the study. A gastrointestinal surgeon and radiologist independently evaluated $20 \mathrm{CT}$ scans of patients following Ivor Lewis esophagectomy, consisting of 10 healthy postoperative patients and 10 patients with known anastomotic leakage. The following CT findings were selected in consensus: extraluminal oral contrast, visible wall discontinuity, air collection at the anastomotic site (located $2 \mathrm{~cm}$ proximal or distal to the anastomosis), mediastinal air collection, air collection below the diaphragm, subcutaneous emphysema, pneumothorax, fluid collection at the anastomotic site (located $2 \mathrm{~cm}$ proximal or distal to the anastomosis), mediastinal fluid collection, loculated pleural effusion (pocket of or trapped pleural fluid) and infiltration of fat at the anastomotic site (non-contiguous inhomogeneity with low tissue density, located $2 \mathrm{~cm}$ proximal or distal to the anastomosis).

\section{Review process}

All CT images were randomized and independently assessed by two experienced gastrointestinal radiologists (A and B), who were blinded for clinical information. The presence or absence of the predetermined CT findings was systematically documented. The radiologists finalized the assessment by stating whether anastomotic leakage was suspected (classified as "no anastomotic leakage" or "suspected anastomotic leakage"), further referred to as "blinded interpretation of the radiologists". Following blinded assessment by the two radiologists, the original CT reports were re-evaluated and conclusions were classified as "no anastomotic leakage", "suspicion of anastomotic leakage" or "definite anastomotic leak".

\section{Anastomotic leakage}

Anastomotic leakage was clinically suspected based on clinical deterioration, elevated serum c-reactive protein and/or abnormal drain production. Clinical deterioration was defined by a combination of tachycardia ( $>100$ beats per minute), tachypnea (ventilation rate of $>20$ per minute), hypotension (systolic pressure $<100 \mathrm{mmHg}$ ) or fever $\left(>38^{\circ} \mathrm{C}\right)$. Serum c-reactive protein levels of $>140 \mathrm{mg} / \mathrm{dL}$ were considered elevated. When anastomotic leakage was clinically suspected, a CT scan was requested to objectify. In this study, leakage was later confirmed by visualization of the defect during endoscopy or thoracotomy. Severity was graded based on required treatment according to definitions stated by the Esophagectomy Complications Consensus Group (ECCG) (18). Additional patient, surgery and pathology data were registered.

\section{Statistical analysis and scoring system}

For continuous data, independent sample t tests or MannWhitney U tests were used. Dichotomous and categorical variables were analyzed using Chi-Square tests or Fisher's exact tests (in case of small cell counts). The level of interobserver agreement between radiologists was derived from Kappa values and defined as follows: $0-0.20$ poor agreement, $0.21-0.40$ fair agreement, $0.41-0.60$ moderate agreement, $0.61-0.80$ substantial agreement and $0.81-1$ excellent agreement. The original report and blinded interpretation of the radiologists were used as reference standard and their diagnostic performances were computed by means of cross tabulation and receiver operating characteristics (ROC). Univariable logistic regression was used to determine the association between individual CT findings and anastomotic leakage, providing odds ratios (OR) with $95 \%$ confidence intervals (CI) and p-values. Subgroup analyses were performed to assess whether timing of CT imaging [postoperative day (POD) 1-4 vs. POD 5-30] influenced the associations of the different CT findings with anastomotic leakage. Multivariable backward stepwise logistic regression of CT findings was performed to develop a CT-based practical scoring system. CT findings with a p-value of $<0.157$, based on the Akaike information criterion (19), retained in the scoring system. Based on the beta-regression coefficients of the retained CT findings, previous research $(13,14)$ and expert opinion the importance of each CT finding was determined and converted into an amount of points. A higher amount of points indicated a higher predicted probability of anastomotic leakage. The diagnostic performance was determined by ROC curve analysis, and calculation of the area under the receiver operating characteristics (AUROC). Sensitivity, specificity, positive predictive value (PPV) and negative predictive value (NPV) were computed for each amount of points. The diagnostic accuracy of the scoring system was compared to the blinded interpretation of the radiologists and original reports (reference standard). Radiologist A had a superior 


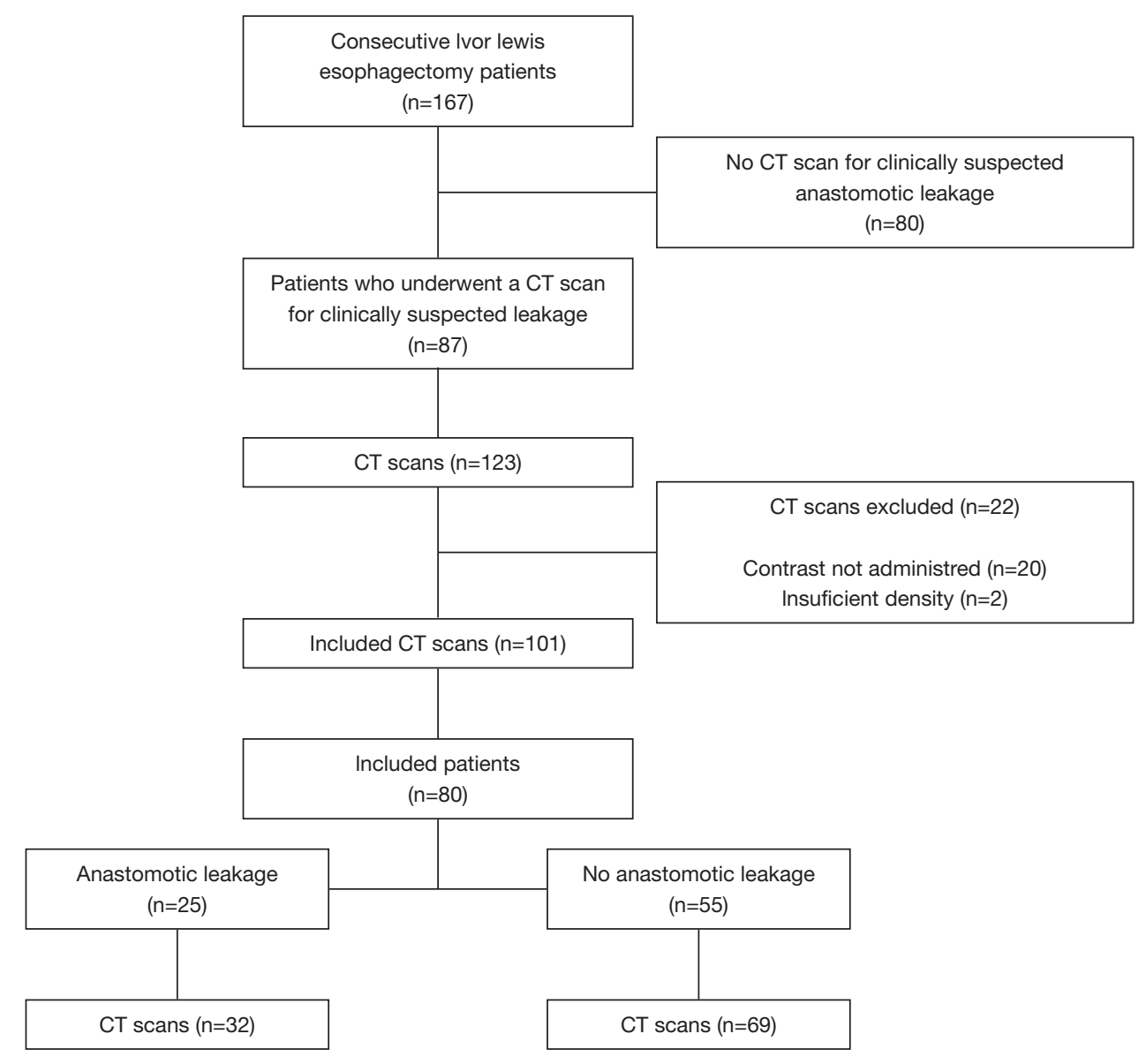

Figure 1 Flowchart shows the selection of the study population.

diagnostic accuracy over radiologist $\mathrm{B}$ and therefore the CT assessment of radiologist A was used for logistic regression analysis. IBM SPSS statistics (version 23) was used for standard statistical analysis and a $\mathrm{P}$ value of $<0.05$ was considered statistically significant.

\section{Results}

\section{Patient characteristics}

In our study population, 167 patients underwent esophagectomy with an intrathoracic anastomosis (Figure 1). Of these patients, 87 had clinical signs of anastomotic leakage and one or multiple CT scans were performed. Seven patients were excluded (22 CT scans) due to insufficient density of oral contrast or oral contrast was omitted, resulting in 80 patients and a total of $101 \mathrm{CT}$ scans (Figure 1). Median POD of the scans was day 4 [inter quartile range (IQR): 3-9]. Further subject characteristics are summarized in Table 1, no statistically significant differences were found between groups.

\section{Anastomotic leakage}

Thirty-two CT scans were performed in 25 patients with a confirmed anastomotic leak. Median POD to clinical diagnosis of anastomotic leakage was day 9 (IQR, 6-12). Initially, leakage was diagnosed by CT imaging (15 patients) or, when required, by additional endoscopy (9 patients), diagnostic thoracotomy (1 patient). Leakage was subsequently confirmed by a therapeutic endoscopy (15 patients) or thoracotomy (10 patients). Endoscopic stent placement was not feasible in one patient and was treated with antibiotic therapy, nasogastric tube drainage and total parenteral nutrition. This resulted in one patient with a grade 1 anastomotic leak, 14 patients with grade 2 
Table 1 Summary of demographic and clinical characteristics

\begin{tabular}{|c|c|c|}
\hline Characteristics & $A L(n=25)$ & Controls $(n=55)$ \\
\hline Male gender & $24(96.0)$ & $50(90.9)$ \\
\hline Age (years) & $64.0(52.5-68.0)$ & $63.5(56.0-70.0)$ \\
\hline \multicolumn{3}{|l|}{ ASA classification } \\
\hline 1 & $3(12.0)$ & $3(5.5)$ \\
\hline 2 & $17(68.0)$ & $37(67.3)$ \\
\hline 3 & $5(20.0)$ & $15(27.3)$ \\
\hline \multicolumn{3}{|l|}{ Type of carcinoma } \\
\hline Adenocarcinoma & $13(52.0)$ & $25(45.5)$ \\
\hline $\begin{array}{l}\text { Squamous cell } \\
\text { carcinoma }\end{array}$ & $12(48.0)$ & $28(50.9)$ \\
\hline Other & $0(0)$ & $2(3.6)$ \\
\hline \multicolumn{3}{|l|}{ Neoadjuvant treatment } \\
\hline Chemoradiotherapy & $20(80.0)$ & $53(96.4)$ \\
\hline Chemotherapy alone & $2(8.0)$ & $0(0)$ \\
\hline None & $3(12.0)$ & $2(3.6)$ \\
\hline $\begin{array}{l}\text { Location tumor } \\
\text { (centimeter) }\end{array}$ & $36.5(33.0-38.5)$ & $35.0(34.0-38.0)$ \\
\hline \multicolumn{3}{|l|}{ T-stage } \\
\hline $\mathrm{T} 1$ & $3(12.0)$ & $2(3.6)$ \\
\hline $\mathrm{T} 2$ & $4(16.0)$ & 7 (12.7) \\
\hline T3 & $17(68.0)$ & $44(80.0)$ \\
\hline $\mathrm{T} 4 \mathrm{a}$ & $1(4.0)$ & $1(1.8)$ \\
\hline Tx & $0(0)$ & $1(1.8)$ \\
\hline $\begin{array}{l}\text { Ivor Lewis } \\
\text { esophagectomy }\end{array}$ & $25(100.0)$ & $55(100.0)$ \\
\hline \multicolumn{3}{|l|}{ Type of anastomosis } \\
\hline ETS Circular stapled & $3(12.0)$ & $6(10.9)$ \\
\hline ETS Linear stapled & $10(40.0)$ & $15(27.3)$ \\
\hline STS Linear stapled & $12(48.0)$ & $34(61.8)$ \\
\hline \multicolumn{3}{|l|}{ Inflammatory markers } \\
\hline $\begin{array}{l}\text { C-reactive protein } \\
(\mathrm{mg} / \mathrm{L})\end{array}$ & 185 [110-302] & $190[135-270]$ \\
\hline $\begin{array}{l}\text { White blood cell count } \\
\left(\times 10^{9} / \mathrm{L}\right)\end{array}$ & $12.4(8.3-16.3)$ & $10.8(8.7-14.0)$ \\
\hline $\begin{array}{l}\text { Removal thoracic drain } \\
\text { (POD) }\end{array}$ & $2[2-4]$ & $3[2-5]$ \\
\hline CT (POD) & $5.5(4.0-8.5)$ & $4.0(3.0-9.0)$ \\
\hline
\end{tabular}

Table 1 (continued)
Table 1 (continued)

\begin{tabular}{|c|c|c|}
\hline Characteristics & $A L(n=25)$ & Controls $(n=55)$ \\
\hline \multicolumn{3}{|l|}{ Number of CT scans } \\
\hline 1 & $18(72.0)$ & $42(76.4)$ \\
\hline 2 & $7(28.0)$ & $12(21.8)$ \\
\hline 3 & $0(0.0)$ & $1(1.8)$ \\
\hline Total & 32 & 69 \\
\hline Diagnosis AL (POD) & 7 [6-12] & - \\
\hline \multicolumn{3}{|c|}{ Grading AL according to ECCG } \\
\hline Grade 1 & $1(4.0)$ & - \\
\hline Grade 2 & $14(56.0)$ & - \\
\hline Grade 3 & $10(40.0)$ & - \\
\hline \multicolumn{3}{|l|}{ Other complications } \\
\hline Wound infection & $1(4.0)$ & $3(5.5)$ \\
\hline Pneumonia & $6(24.0)$ & $26(47.3)$ \\
\hline \multicolumn{3}{|l|}{ Additional treatment } \\
\hline Nil per os & $23(92.0)$ & $18(33.3)$ \\
\hline Antibiotics & $25(100)$ & $34(61.8)$ \\
\hline $\begin{array}{l}\text { Percutaneous thoracic } \\
\text { drainage }\end{array}$ & $6(24.0)$ & $3(5.5)$ \\
\hline Additional endoscopies* & $10(40.0)$ & $24(43.6)$ \\
\hline
\end{tabular}

Data are $\mathrm{n}(\%)$ or median (IQR). *, performed prior to diagnosis of anastomotic leakage. AL, anastomotic leakage; ASA, American Society of Anesthesiologists; CT, computed tomography; ECCG, Esophagectomy Complications Consensus Group; ETS, end-toside; POD, postoperative day; STS, side-to-side; T-stage, tumor stage.

anastomotic leaks and finally 10 patients with a grade 3 leak [grading according to ECCG (18)].

\section{Multiple CT scans}

In 20 patients multiple CT scans [two CT scans $(n=19)$ or three CT scans $(n=1)$ ], were required to diagnose or rule out anastomotic leakage. The first scans (median POD 4) reported no anastomotic leak $(n=18)$ or suspected leak $(n=2)$. Seven patients underwent an additional endoscopy in which no leaks were seen. All patients were closely monitored and when the suspicion of anastomotic leakage remained, a second CT scan was requested to identify new findings (median POD 12). The second CT scan concluded a clear 
Table 2 Inter-observer variability between radiologist A and radiologist B for each CT finding

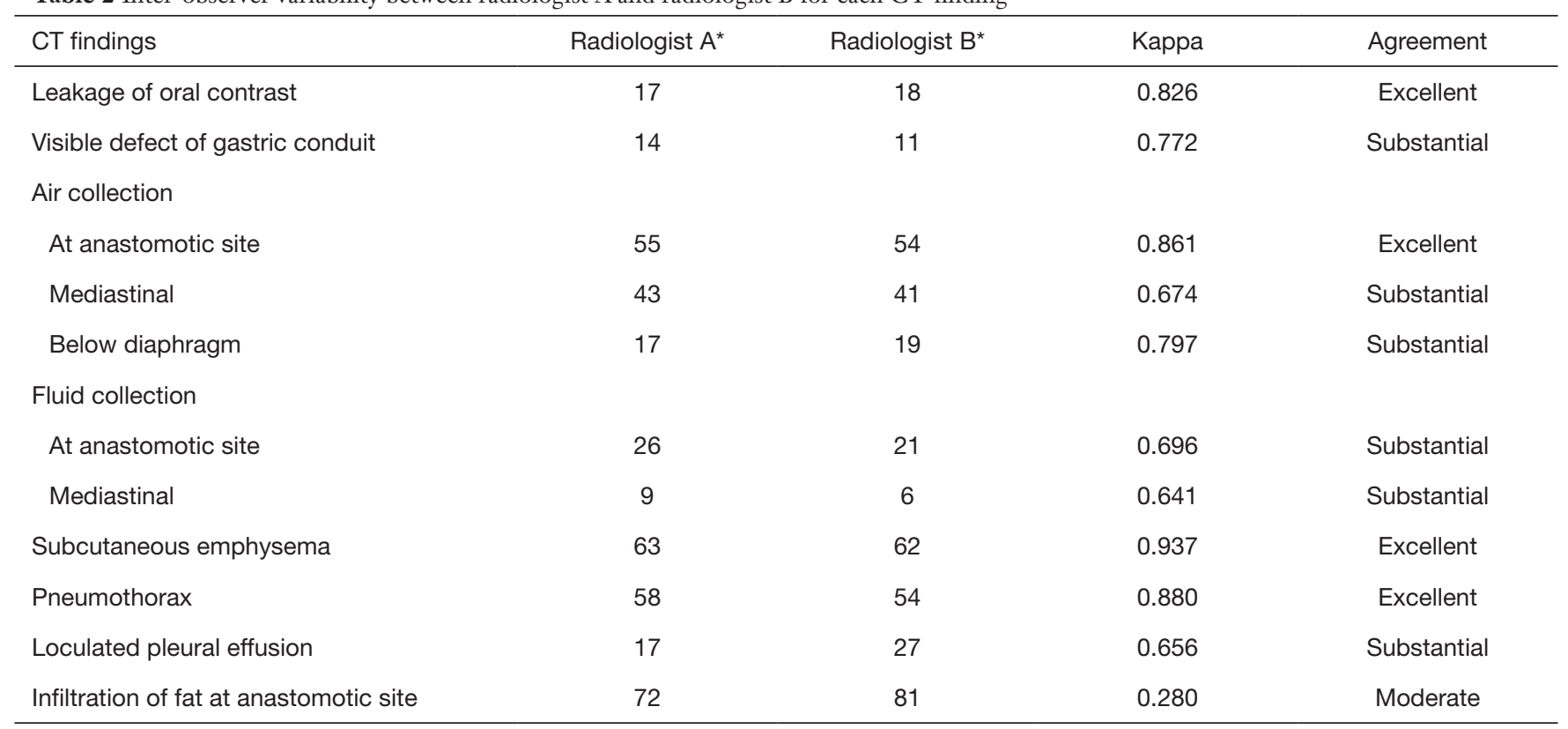

${ }^{*}$, number of scans in which the CT finding was present. CT, computed tomography.

anastomotic leak $(n=4)$, suspected leak $(n=3)$ or no leak $(\mathrm{n}=13)$. Additional diagnostic endoscopies were performed in 8 patients, which revealed an anastomotic leak in three patients, and no apparent leak in five patients. In one patient a third CT scan (POD 22) reported a suspected leak, however additional endoscopy revealed no leak in this patient.

\section{Inter-observer agreement}

The results of the Cohen's kappa measures for both radiologists are displayed in Table 2. Overall, the interobserver agreement between the two radiologists was substantial to excellent for most CT findings. The interobserver agreement of the presence of surrounding tissue infiltration was moderate with a Kappa value of 0.280 . The rest of the findings had a Kappa value of 0.641 or more.

\section{Predictive CT findings}

The individual CT findings and their association with postoperative anastomotic leakage are displayed in Table 3. Univariable logistic regression analysis showed that the presence of extraluminal oral contrast (OR: 19.41, 95\% CI: 5.04-74.83, $\mathrm{P}<0.001$ ), visible wall discontinuity (OR: 5.01, 95\% CI: $1.52-16.51, \mathrm{P}=0.008)$, air collection at the anastomotic site (OR: 10.89, 95\% CI: 3.44-34.52, $\mathrm{P}<0.001$ ), mediastinal air collection (OR: 5.03, 95\% CI: 2.03-12.45, $\mathrm{P}<0.001)$, fluid collection at the anastomotic site (OR: 3.69, 95\% CI: 1.45-9.42, $\mathrm{P}=0.006)$, mediastinal fluid collection (OR: 5.08, 95\% CI: 1.18-21.82, $\mathrm{P}=0.029$ ), pneumothorax (OR: 3.09, 95\% CI: 1.22-7.82, $\mathrm{P}=0.017$ ), loculated pleural effusion (OR: $5.50,95 \% \mathrm{CI}: 1.81-16.70, \mathrm{P}=0.003)$ and infiltration of fat at anastomotic site (OR: $5.85,95 \%$ CI: $1.62-21.12, \mathrm{P}=0.007)$ were significantly associated with the occurrence of anastomotic leakage.

Subgroup analysis was performed for early CT scans (POD 1-4) and late CT scans (POD 5-30). The early group contained 54 scans, anastomotic leakage was confirmed in 16 scans and the late group consisted of 47 scans in which a leak was confirmed in $16 \mathrm{CT}$ scans. These differences were not significant $(\mathrm{P}=0.634)$. In patients without anastomotic leakage subcutaneous emphysema ( $81.6 \%$ vs. $41.9 \%$, $\mathrm{P}=0.001)$ and air collections below the diaphragm $(26.3 \%$ vs. $0.0 \%, \mathrm{P}=0.002$ ) were more frequently observed in early compared to late scans. No significant differences in CTscan findings were observed between early and late CTscans in patients that suffered anastomotic leakage.

\section{Scoring system}

Multivariable backward stepwise logistic regression analysis 
Table 3 Summary of univariable logistic regression analysis of separate CT findings

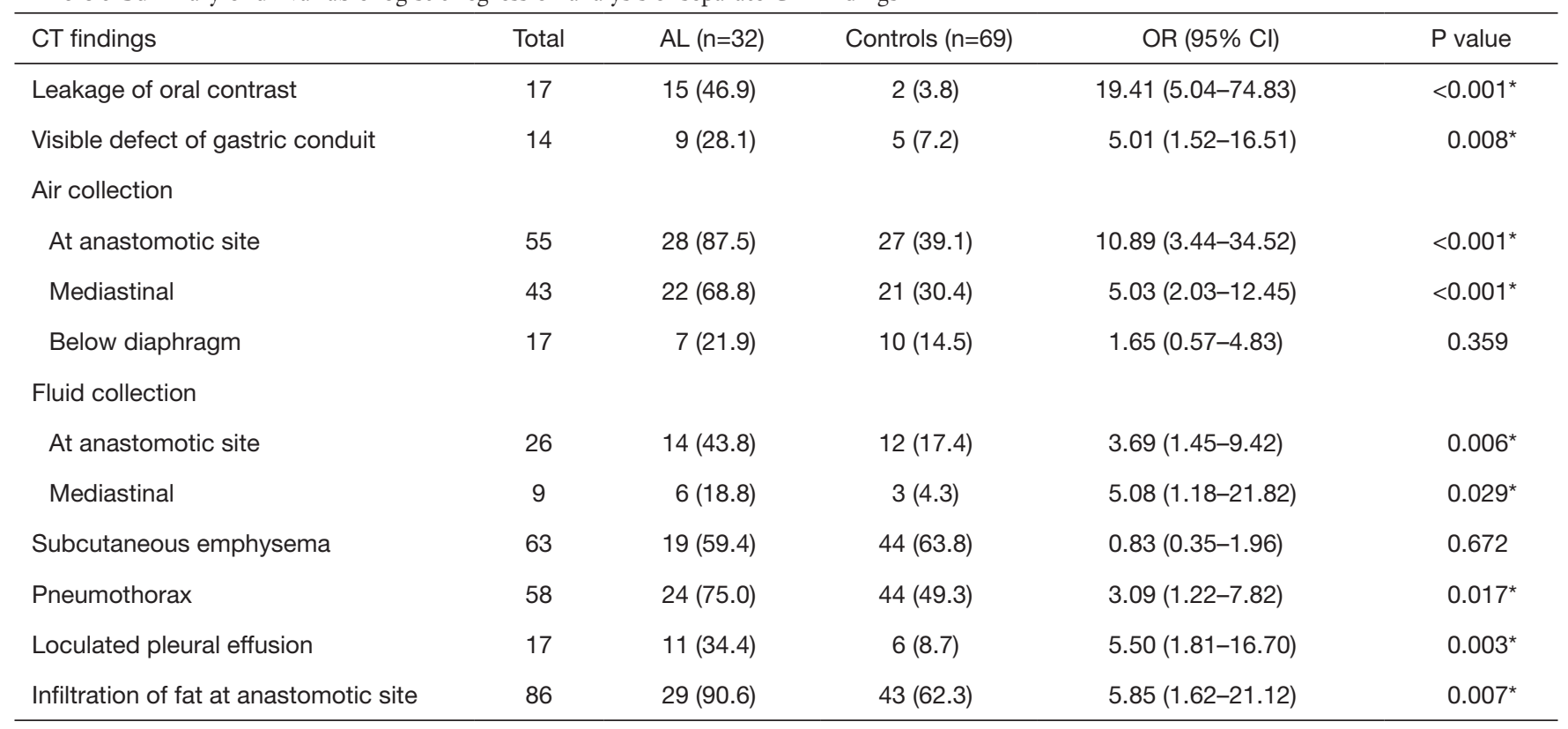

Data are $\mathrm{n}(\%) .{ }^{*}$, statistically significant association between CT parameter and anastomotic leakage. AL, anastomotic leakage; CT, computed tomography.

Table 4 Diagnostic accuracy of the initial interpretation of the radiologist and the scoring system. Scores range from zero to five points and sensitivity, specificity, PPV and NPV of each score is provided

\begin{tabular}{|c|c|c|c|c|}
\hline & $\begin{array}{c}\text { Sensitivity } \\
(\%)\end{array}$ & $\begin{array}{c}\text { Specificity } \\
(\%)\end{array}$ & $\begin{array}{l}\text { PPV } \\
(\%)\end{array}$ & $\begin{array}{l}\text { NPV } \\
(\%)\end{array}$ \\
\hline \multicolumn{5}{|c|}{ Initial interpretation } \\
\hline Original report & 68.8 & 88.4 & 73.3 & 85.9 \\
\hline Radiologist A & 75.0 & 85.5 & 70.6 & 88.1 \\
\hline Radiologist B & 59.4 & 85.5 & 65.5 & 81.9 \\
\hline \multicolumn{5}{|l|}{ Scoring system } \\
\hline 0 points & 100.0 & 0.0 & 31.7 & - \\
\hline 1 point & 100.0 & 24.6 & 38.1 & 100.0 \\
\hline 2 points & 90.6 & 63.8 & 53.7 & 93.6 \\
\hline 3 points & 62.5 & 94.2 & 83.3 & 84.4 \\
\hline 4 points & 28.1 & 98.6 & 90.0 & 74.7 \\
\hline 5 points & 6.3 & 100.0 & 100.0 & 69.7 \\
\hline
\end{tabular}

PPV, positive predictive value; NPV, negative predictive value.

was performed and all individual CT findings were included in the initial analysis. After multiple rounds the following findings retained in the model: presence of extraluminal oral contrast ( $\beta$ coefficient: 1.688 , OR: $5.41,95 \%$ CI: $1.13-25.79, \mathrm{P}=0.034)$, air collection at the anastomotic site $(\beta$ coefficient: 1.744, OR: 5.72 , 95\% CI: $1.46-22.41, \mathrm{P}=0.012$ ), fluid collection at the anastomotic site $(\beta$ coefficient: 1.434 , OR: 4.20, 95\% CI: 1.08-16.38, $\mathrm{P}=0.039)$, pneumothorax $(\beta$ coefficient: 1.841 , OR: $6.30,95 \%$ CI: $1.55-25.62, \mathrm{P}=0.010$ ), and loculated pleural effusion ( $\beta$ coefficient: 2.336 , OR: 10.34, 95\% CI: $1.53-70.07, \mathrm{P}=0.017)$. It was decided that all characteristics were given one point each (Table 4, Figure 2), resulting in a cumulative score ranging from 0 to 5 . The 5 -point scoring system was applied to the entire dataset, resulting in an observed AUROC of 0.875 (95\% CI: 0.802 0.947). The ROC was compared to the original report and blinded interpretation of the radiologists and represented in Figure 3. The sensitivity, specificity, PPV and NPV for each score is displayed in Table 5. A score of 3 or more points was statistically determined as the optimal cut-off and yielded a sensitivity of $62.5 \%$, specificity of $94.2 \%$, PPV of $83.3 \%$ and NPV of $84.4 \%$.

\section{Diagnostic value of CT imaging}

The original CT reports concluded no anastomotic leakage in 71 scans, of which 10 had a confirmed anastomotic leak (NPV: $85.9 \%$ ). An anastomotic leak was suspected in 30 

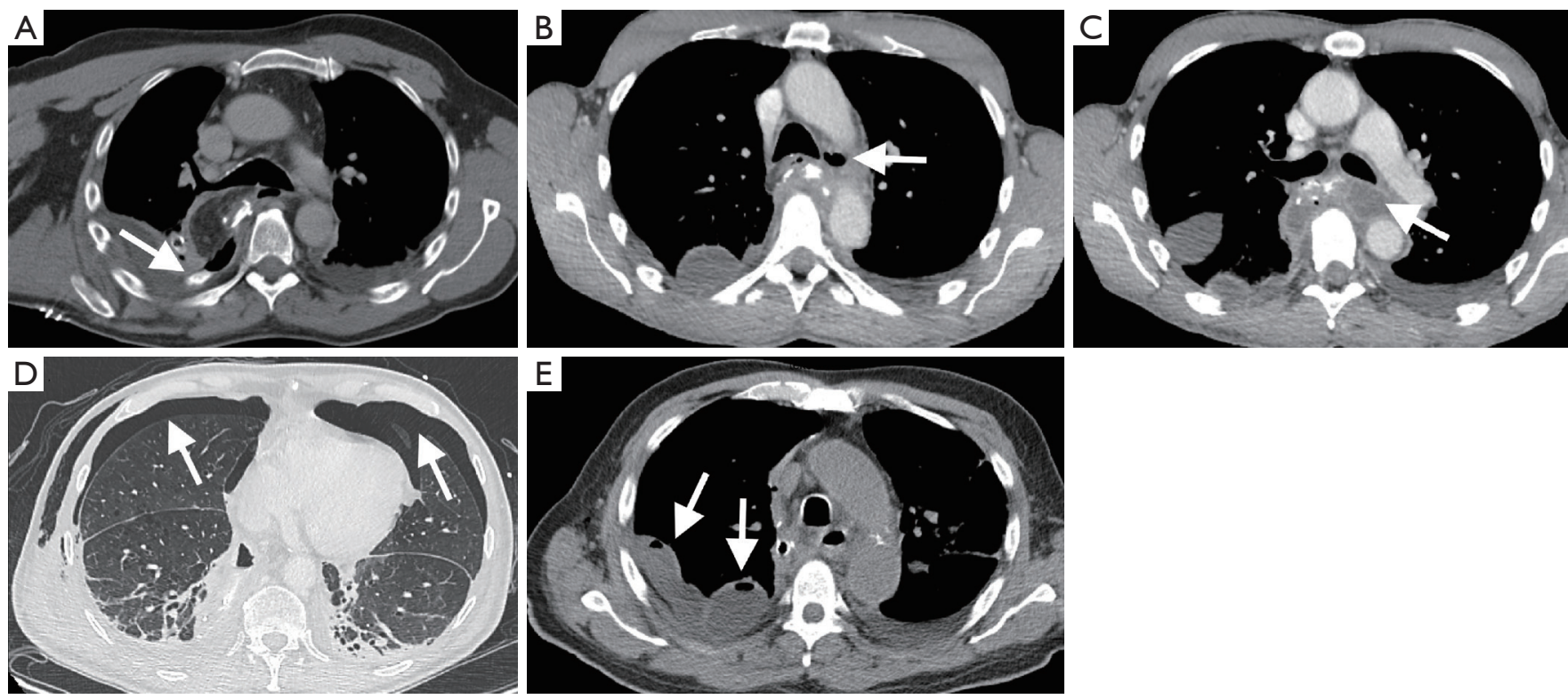

Figure 2 Examples of specific CT findings (arrows) included in the scoring system following Ivor Lewis esophagectomy. (A) Image shows leakage of oral contrast out of the gastric conduit. (B) Image shows extraluminal air collection at the anastomotic site. (C) Image shows fluid collection at the anastomotic site. (D) Image shows a bilateral pneumothorax. (E) Image shows loculated pleural effusion. CT, computed tomography.

scans (16 definite leaks and 14 suspected leaks), which was confirmed in 22 cases (PPV: $73.3 \%$ ). This resulted in an AUROC of 0.786 (95\% CI: 0.680-0.891) and a corresponding sensitivity of $68.8 \%$ and specificity of $88.4 \%$. Radiologists A and B suspected anastomotic leakage in 34 and 29 scans, which appeared to be the case in 24 and 19 scans (PPV: $70.6 \%$ and $65.5 \%$ respectively). The absence of leakage was scored in 67 and 72 scans of which 8 and 13 had a confirmed leak (NPV: $88.1 \%$ and $81.9 \%$ respectively). This resulted in AUROC of 0.803 (95\% CI: 0.702-0.903) for radiologist A (sensitivity: $75.0 \%$ and specificity: $85.5 \%$ ) and 0.724 (95\% CI: 0.610-0.724) for radiologist B (sensitivity: $59.4 \%$ and specificity: $85.5 \%$ ). The diagnostic performance of the original report and blinded interpretation of the radiologists are summarized in Table 5, the corresponding ROC curve analyses are displayed in Figure 3.

\section{Discussion}

This study reported the diagnostic accuracy of CT imaging in patients who were clinically suspected of having a postoperative anastomotic leak. A sensitivity ranging from $59.4 \%$ to $75.0 \%$ and a specificity ranging from $85.5 \%$ to $88.4 \%$ was observed. Additionally, CT findings were systematically analyzed and predictors of anastomotic leakage were identified. This resulted in a practical 5-point scoring system involving the following CT findings: extraluminal oral contrast, air collection at the anastomotic site, fluid collection at the anastomotic site, pneumothorax and loculated pleural effusion. A score of 3 points was determined as optimal cut-off, in which patients with 3 or more points were considered at high risk of anastomotic leakage, warranting additional endoscopic evaluation to confirm the diagnosis, evaluate the size of the defect or initiate early treatment. Patients with 2 or even less points were considered at low risk for anastomotic leakage, for these patients a wait and see strategy is justified.

To date, this study is the largest of two studies investigating individual CT findings for the detection of intrathoracic anastomotic leakage. Upponi et al. (20) showed that air and fluid collections in the mediastinum had a predictive value in the assessment of anastomotic leakage. Strauss et al. (21) observed that the presence of contrast leakage and mediastinal air collections were associated with anastomotic leakage. The current study not only confirms the data in a larger cohort, but developed a clinically applicable scoring system for the detection of 


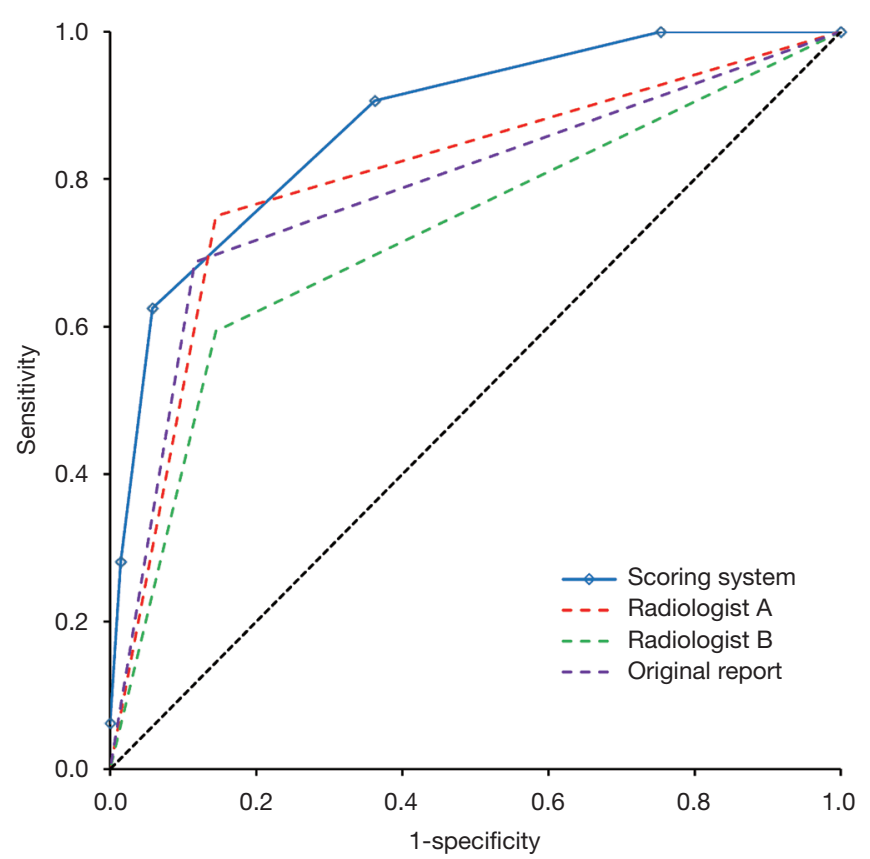

Figure 3 ROC analysis of the scoring system (blue line), the blinded interpretation by radiologist A (red dotted line), blinded interpretation by radiologist B (green dotted line) and original report (purple dotted line). Results indicate improved predictive abilities of the scoring system compared to the blinded interpretation of the radiologist and original reports. Receiver operating characteristic (ROC).

Table 5 CT findings included in the scoring system

\begin{tabular}{lc}
\hline CT findings & Points \\
\hline Leakage of oral contrast & 1 \\
Air collection at the anastomotic site & 1 \\
Fluid collection at the anastomotic site & 1 \\
Pneumothorax & 1 \\
Loculated pleural effusion & 1 \\
$\geq 3$ points: high probability of anastomotic & \\
leakage, treatment warranted &
\end{tabular}

$\mathrm{CT}$, computed tomography.

anastomotic leakage. Goense et al. (13) made a similar effort to develop a CT-based risk score for cervical leaks. Cervical leakage is associated with other CT findings compared to intrathoracic leaks and was therefore not our interest. In current study, the presence of extraluminal oral contrast, loculated pleural effusion and anastomotic or mediastinal air/fluid collections were registered.

Besides CT imaging with oral contrast, routine contrast swallow radiography has been suggested to assess anastomotic integrity. Multiple studies have been conducted demonstrating similar specificities for both examinations. Overall, CT imaging is associated with a higher sensitivity compared to radiography (20-22). Additionally, CT imaging has the added benefit of being easier to perform in critically ill patients, allows for recognition of associated findings (e.g., pleural effusion, pneumothorax, pulmonary abnormalities or undrained fluid collections) and can be performed by radiologic technologists. On the other hand, the obligatory delay in CT scanning following oral contrast administration could lead to increased false negative results for subclinical grade 1 leaks, compared to real-time radiography.

Endoscopic evaluation of the anastomosis provides excellent visibility of the surgical site and remains the golden standard in case of inconclusive imaging (23). On the other hand, aspiration, iatrogenic injuries and deterioration of the anastomotic dehiscence by inflating the esophagus remain a concern. When clinical findings are indicative for anastomotic leakage, CT imaging is justified as first diagnostic approach, followed by endoscopic evaluation in case of uncertainty.

In this study, the inter-observer agreement between the radiologists proved to be substantial to excellent for the findings included in the scoring system, yet there was a difference in their interpretation. The accuracy for predicting leakage of radiologist A was substantially better compared to radiologist B (AUROC: 0.803 and 0.724 respectively), indicating that, even when assessment of individual CT findings is accurate, interpretation remains challenging. As a result, 10 out of 25 patients with anastomotic leakage required an additional endoscopy or thoracotomy to diagnose the anastomotic leak. The proposed scoring system could serve as a guide for radiologists and surgeons in the systematic assessment of CT scans and prediction of anastomotic leakage. Further validation is needed to solidify its diagnostic accuracy amongst other radiologists and surgeons.

After multivariable analysis, loculated pleural effusion was the strongest predictor for anastomotic leakage, indicated by its superior beta regression coefficient. Extraluminal oral contrast showed to be the strongest individual predictor. Yet, both findings received one point in the scoring system. Assigning 2 or more points to the presence of extraluminal oral contrast or loculated pleural effusion did not improve 
the discriminative ability of the scoring system. This can be attributed to the observation that both findings were hardly reported without the presence of multiple other findings included in the scoring system.

This study is subject to potential limitations. First of all, some subclinical grade 1 leaks might have been missed as only not all patients underwent a postoperative endoscopy in that group. Furthermore, CT imaging might be less effective in detecting small grade 1 leaks due to the obligatory delay between scanning and oral contrast administration. Second, most patients with anastomotic leakage in our cohort underwent either a therapeutic endoscopy or thoracotomy (grade 2 and 3), which makes it difficult to extrapolate the diagnostic accuracy of the scoring system to early grade 1 anastomotic leaks. Finally, the scoring system needs to be externally validated by different radiologists on another cohort of patients.

Literature shows that laboratory findings of increased inflammatory markers can be predictive for anastomotic leakage $(24,25)$. Imputing these with CT findings other factors, such as clinical symptoms and vital parameters, could render a diagnostic algorithm with a very high posttest probability. Further prospective research should focus on developing such an algorithm and determine its clinical value by comparing it to current practice.

In conclusion, this study provides an easy to use 5-point scoring system with good diagnostic accuracy and low interobserver variability for the assessment of the anastomotic integrity after Ivor Lewis esophagectomy. A score of 3 or more points indicated a high probability of anastomotic leakage, justifying early initiation of treatment or additional endoscopic evaluation. Patients with lower scores are considered at low risk for anastomotic leakage, however vigilant assessment remains warranted.

\section{Acknowledgments}

Funding: None.

\section{Footnote}

Reporting Checklist: The authors have completed the STROBE reporting checklist. Available at http://dx.doi. org/10.21037/jtd-20-954

Data Sharing Statement: http://dx.doi.org/10.21037/jtd20-954
Conflicts of Interest: All authors have completed the ICMJE uniform disclosure form (available at http://dx.doi. org/10.21037/jtd-20-954). The authors have no conflicts of interest to declare.

Ethical Statement: The authors are accountable for all aspects of the work in ensuring that questions related to the accuracy or integrity of any part of the work are appropriately investigated and resolved. This study was conducted in accordance with the Declaration of Helsinki (as revised in 2013). The study protocol was approved by the Medical Ethical Review Committee of the VU University Medical Center (2016.480) and by the local institutional ethical review board of the Catharina Hospital.

Open Access Statement: This is an Open Access article distributed in accordance with the Creative Commons Attribution-NonCommercial-NoDerivs 4.0 International License (CC BY-NC-ND 4.0), which permits the noncommercial replication and distribution of the article with the strict proviso that no changes or edits are made and the original work is properly cited (including links to both the formal publication through the relevant DOI and the license). See: https://creativecommons.org/licenses/by-nc-nd/4.0/.

\section{References}

1. Ferlay J, Soerjomataram I, Dikshit R, et al. Cancer incidence and mortality worldwide: sources, methods and major patterns in GLOBOCAN 2012. Int J Cancer 2015;136:E359-86.

2. van Hagen $\mathrm{P}$, Hulshof MC, van Lanschot JJ, et al. Preoperative chemoradiotherapy for esophageal or junctional cancer. N Engl J Med 2012;366:2074-84.

3. Thrift AP. The epidemic of oesophageal carcinoma: Where are we now? Cancer Epidemiol 2016;41:88-95.

4. Biere SS, Maas KW, Cuesta MA, et al. Cervical or thoracic anastomosis after esophagectomy for cancer: a systematic review and meta-analysis. Dig Surg 2011;28:29-35.

5. Goense L, Meziani J, Ruurda JP, et al. Impact of postoperative complications on outcomes after oesophagectomy for cancer. Br J Surg 2019;106:111-9.

6. Gooszen JAH, Goense L, Gisbertz SS, et al. Intrathoracic versus cervical anastomosis and predictors of anastomotic leakage after oesophagectomy for cancer. The $\mathrm{Br} \mathrm{J}$ Surg 2018;105:552-60.

7. Rizk NP, Bach PB, Schrag D, et al. The impact of 
complications on outcomes after resection for esophageal and gastroesophageal junction carcinoma. J Am Coll Surg 2004;198:42-50.

8. Markar S, Gronnier C, Duhamel A, et al. The Impact of Severe Anastomotic Leak on Long-term Survival and Cancer Recurrence After Surgical Resection for Esophageal Malignancy. Ann Surg 2015;262:972-80.

9. Turrentine FE, Denlinger CE, Simpson VB, et al. Morbidity, mortality, cost, and survival estimates of gastrointestinal anastomotic leaks. J Am Coll Surg 2015;220:195-206.

10. Goense L, van Dijk WA, Govaert JA, et al. Hospital costs of complications after esophagectomy for cancer. Eur J Surg Oncol 2017;43:696-702.

11. Moon SW, Kim JJ, Cho DG, et al. Early detection of complications: anastomotic leakage. J Thorac Dis 2019;11:S805-11.

12. Messager M, Warlaumont M, Renaud F, et al. Recent improvements in the management of esophageal anastomotic leak after surgery for cancer. Eur J Surg Oncol 2017;43:258-69.

13. Goense L, Stassen PM, Wessels FJ, et al. Diagnostic performance of a CT-based scoring system for diagnosis of anastomotic leakage after esophagectomy: comparison with subjective CT assessment. Eur Radiol 2017;27:4426-34.

14. Power N, Atri M, Ryan S, et al. CT assessment of anastomotic bowel leak. Clin Radiol 2007;62:37-42.

15. Kim TH, Kim JH, Shin CI, et al. CT findings suggesting anastomotic leak and predicting the recovery period following gastric surgery. Eur Radiol 2015;25:1958-66.

16. Lewis I. The surgical treatment of carcinoma of the oesophagus; with special reference to a new operation for growths of the middle third. Br J Surg 1946;34:18-31.

17. von Elm E, Altman DG, Egger M, et al. Strengthening the Reporting of Observational Studies in Epidemiology (STROBE) statement: guidelines for reporting

Cite this article as: Plat VD, Bootsma BT, Straatman J, van den Bergh J, van Waesberghe JHTM, Luttikhold J, Luyer MDP, van der Peet DL, Daams F. The clinical suspicion of a leaking intrathoracic esophagogastric anastomosis: the role of CT imaging. J Thorac Dis 2020;12(12):7182-7192. doi: 10.21037/ jtd-20-954 observational studies. BMJ 2007;335:806-8.

18. Low DE, Alderson D, Cecconello I, et al. International Consensus on Standardization of Data Collection for Complications Associated With Esophagectomy: Esophagectomy Complications Consensus Group (ECCG). Ann Surg 2015;262:286-94.

19. Heinze G, Wallisch C, Dunkler D. Variable selection - A review and recommendations for the practicing statistician. Biom J 2018;60:431-49.

20. Upponi S, Ganeshan A, D'Costa H, et al. Radiological detection of post-oesophagectomy anastomotic leak - a comparison between multidetector CT and fluoroscopy. Br J Radiol 2008;81:545-8.

21. Strauss C, Mal F, Perniceni T, et al. Computed tomography versus water-soluble contrast swallow in the detection of intrathoracic anastomotic leak complicating esophagogastrectomy (Ivor Lewis): a prospective study in 97 patients. Ann Surg 2010;251:647-51.

22. Hogan BA, Winter DC, Broe D, et al. Prospective trial comparing contrast swallow, computed tomography and endoscopy to identify anastomotic leak following oesophagogastric surgery. Surg Endosc 2008;22:767-71.

23. Schaible A, Sauer P, Hartwig W, et al. Radiologic versus endoscopic evaluation of the conduit after esophageal resection: a prospective, blinded, intraindividually controlled diagnostic study. Surg Endosc 2014;28:2078-85.

24. Aiolfi A, Asti E, Rausa E, et al. Use of C-reactive protein for the early prediction of anastomotic leak after esophagectomy: Systematic review and Bayesian metaanalysis. PLoS One 2018;13:e0209272.

25. Straatman J, Cuesta MA, Schreurs WHH, et al. The PRECious trial PREdiction of Complications, a stepup approach, CRP first followed by CT-scan imaging to ensure quality control after major abdominal surgery: study protocol for a stepped-wedge trial. Trials 2015;16:382. 